\title{
IMMUNOCYTOCHEMICAL STUDIES OF ACTIN LOCALIZATION IN THE CENTRAL NERVOUS SYSTEM ${ }^{1}$
}

\author{
JAMES E. GOLDMAN \\ Department of Pathology (Neuropathology), Albert Einstein College of Medicine, The Bronx, New York 10461
}

Received January 4, 1983; Revised April 19, 1983; Accepted April 28; 1983

\begin{abstract}
The contractile protein, actin, is a major component of the central nervous system (CNS). Its accumulation at presynaptic and postsynaptic sites has been suggested by biochemical and ultrastructural studies. The present work examines directly the distribution of actin within the CNS by immunocytochemical means. Sections of rat CNS were stained with an antiserum against chicken gizzard actin, using a peroxidase-antiperoxidase technique. In Vibratome sections, reaction product appeared widely distributed in the perikaryal cytoplasm of neurons, in axons and dendrites, and in astrocytes and blood vessels. In paraffin sections, however, much of the cytoplasmic reaction was not observed. Instead, the reaction product was concentrated in processes within the neuropil and in discrete densities about the perimeters of neurons. This latter distribution, observed in areas in which large axosomatic synapses are known to occur, was consistent with a concentration at synaptic sites. Immunocytochemistry performed at the ultrastructural level, using a pre-embedding technique, revealed a diffuse cytoplasmic staining, with increased accumulation under the neuronal plasma membrane, particularly at postsynaptic densities.
\end{abstract}

A number of studies over the past several years have demonstrated that actin is a major protein component of the central nervous system (CNS) (Puszkin and Berl, 1972; Bray and Thomas, 1976; Marotta et al., 1978). Although actin is likely to be found in all neurons, there is evidence that its distribution within neurons may not be homogeneous. Studies using subcellular fractionation or histochemical techniques have suggested an accumulation of actin at synaptic sites. For example, actin is a prominent component of fractions enriched in synaptosomes (Puszkin et al., 1972; Blitz and Fine, 1974) and in postsynaptic densities (Cohen et al., 1977; Kelly and Cotman, 1978; Matus and Taff-Jones, 1978). The presence of actin filaments in synaptic regions is also indicated by ultrastructural studies performed with a myosin subfragment labeling technique (Metuzals and Mushynski, 1974; LeBeaux and Willemot, 1975; Alonso et al.,

\footnotetext{
${ }^{1}$ This work was supported in part by National Institutes of Health Grant NS-17125 and by National Institute of Neurological and Communicative Disorders and Stroke Teacher-Investigator Award NS00524. I am grateful to Mr. Philip Lu and Dr. Arthur Vogel of the University of Washington School of Medicine for the anti-actin antiserum, to Ms. Yvonne Kress, Mrs. Dalila Antonetty, and Dr. David Armstrong for advice and assistance with the ultrastructural studies, to Mr. John Katt for technical assistance, to Mrs. Marion Levine and Ms. Renee Sasso for secretarial help, and to Dr. Armstrong and Dr. William Norton for comments on the manuscript.
}

1981; Fifkova and Delay, 1982). In skeletal muscle, actin appears concentrated at neuromuscular junctions (Hall et al., 1981). Previous studies localizing actin within the CNS with immunofluorescent methods have provided evidence for high concentrations of actin in cerebellar glomeruli (Toh et al., 1976), astrocytes (Groschel-Stewart et al., 1977), and blood vessels (Owman et al., 1978). The present report examines the distribution of actin within the CNS using a peroxidase-antiperoxidase (PAP) method applied to tissue sections. These studies support the idea that actin is distributed widely throughout the CNS and throughout the neuronal cytoplasm but is concentrated beneath the neuronal plasma membrane, particularly at postsynaptic sites.

\section{Materials and Methods}

Adult Lewis rats were anesthetized with ether and perfused with $4 \%$ buffered paraformaldehyde. The brains and spinal cords were removed and cut into $2-$ to $3-\mathrm{mm}$ slices. For immunocytochemistry at the light microscopic level two methods of tissue preparation were used. (1) Tissue slices were dehydrated and embedded in paraffin. Immunocytochemistry was performed on $7-\mu \mathrm{m}$-thick sections mounted on gelatin-coated slides, after the sections had been rehydrated. (2) Tissue slices were further fixed in $4 \%$ paraformaldehyde containing $0.1 \%$ glutaraldehyde for $2 \mathrm{hr}$ at room temperature. After washing in phosphate 
buffer at $4^{\circ} \mathrm{C}$ overnight, tissue slices were mounted and 20 - to $25-\mu \mathrm{m}$-thick sections were cut with a Vibratome.

Binding of a rabbit anti-actin antiserum to both paraffin and Vibratome sections was visualized using a peroxidase-antiperoxidase (PAP) technique (Sternberger, 1979). The following series of reactions was carried out at room temperature in a humidified atmosphere: incubation of sections with $3 \% \mathrm{H}_{2} \mathrm{O}_{2}, 5 \mathrm{~min} ; 30 \%$ normal swine serum in phosphate-buffered saline (PBS), $15 \mathrm{~min}$; rabbit anti-actin antiserum (1:250 or 1:500), normal swine serum (1:100) in PBS, 45 min; swine anti-rabbit immunoglobulin (1:20), $30 \mathrm{~min}$; rabbit PAP complex $(1: 50), 30 \mathrm{~min}$; and diaminobenzidine (DAB; $0.05 \%$; Sigma Chemical Co., St. Louis, $\mathrm{MO}), \mathrm{H}_{2} \mathrm{O}_{2}(0.03 \%)$, in $0.1 \mathrm{~m}$ Tris- $\mathrm{HCl}, \mathrm{pH} 7.6,8 \mathrm{~min}$. Incubation of Vibratome sections for $30 \mathrm{~min}$ with sodium borohydride $(10 \mathrm{mg} / \mathrm{ml}$ in PBS) before immunocytochemical staining, to reduce free aldehyde residues, had no effect upon the results with either nonimmune or immune sera. The anti-actin antiserum was raised against chicken gizzard actin, isolated according to the method of Herman and Pollard (1979), by Mr. Philip Lu, University of Washington School of Medicine, and was a gift from Mr. Lu and Dr. Arthur Vogel. Other serum reagents were purchased from DAKO Corp., through Accurate Chemicals, Westbury, NY. Some of the sections were counterstained with hematoxylin. Sections were examined and photographed with a Zeiss photomicroscope equipped with Nomarski optics.

For immunocytochemistry at the ultrastructural level, Vibratome sections were prepared as described above. The PAP reaction was also performed as described above, except that the primary antiserum was used at a 1:500 dilution and sections were incubated with the anti-actin serum for either 45 min or overnight on a shaker at room temperature. Similar results were obtained with either the shorter or longer incubations, except that overnight incubations produced a low level background staining with the nonimmune serum, not seen with the short incubations. After development with $\mathrm{DAB}$, sections were exposed to $1 \%$ osmium tetroxide for $1 \mathrm{hr}$ and embedded in Epon. The distribution of reaction could be visualized at this stage, and appropriate areas were selected for ultrastructural examination. The sections were cut either perpendicular to the plane of the section or obliquely to it and were either not counterstained with heavy metals or stained for 2 min with a $2 \%$ solution of uranyl acetate and examined with a Siemans 101 electron microscope.

The anti-actin antiserum proved to be specific by several criteria. (1) Nonimmune serum gave no reaction product with either paraffin or Vibratome sections of CNS (Fig. 2e). (2) Antiserum was adsorbed with rat brain actin, isolated using DNase I affinity chromatography (Lindberg and Eriksson, 1971; Marotta et al., 1978) (Fig. 1). The antiserum was diluted 1:500 in $1 \mathrm{ml}$ of PBS containing $50 \mu \mathrm{g}$ of rat brain actin; after $1 \mathrm{hr}$ at room temperature, the serum was centrifuged at $100,000 \times g$ for $1 \mathrm{hr}$ at $4^{\circ} \mathrm{C}$. The resulting supernatant produced no reaction product with tissue sections (Fig. 3e). (3) The antiserum reacted with myofibrils of skeletal muscle, smooth muscle of blood vessel walls, and stress fibers of cultured rat astrocytes by immunocytochemical tech-
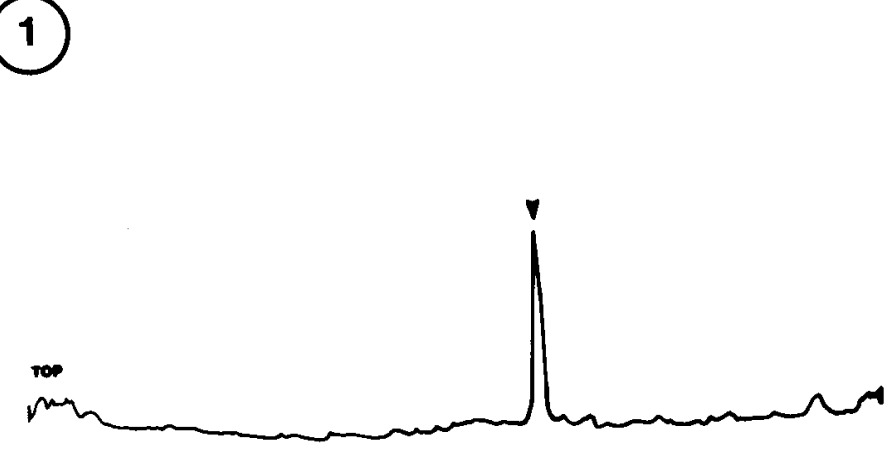

Figure 1. Polyacrylamide gel profile of the rat CNS actin (arrowhead) used for antibody adsorption. Actin, isolated from rat CNS by DNase I affinity chromatography, was electrophoresed on a 5 to $15 \%$ gradient acrylamide gel in sodium dodecyl sulfate (Goldman et al., 1978); the gel was stained with Coomassie blue and scanned at $560 \mathrm{~nm}$. Fifteen micrograms of protein were loaded on the gel.

niques (not shown). Furthermore, when rat astrocyte proteins were separated by polyacrylamide gel electrophoresis and antigen-antibody binding performed directly on gel slices, the antiserum reacted only with a protein of 42,000 daltons (Goldman and Chiu, 1983), previously shown to be actin (Chiu et al., 1981).

\section{Results}

\section{Light microscopy}

Vibratome sections. Results with Vibratome sections and with paraffin sections will be described separately, since the pattern of staining depended upon the way tissues were prepared. In Vibratome sections the reaction product appeared widely distributed throughout the CNS. Neurons displayed a diffuse cytoplasmic staining pattern in perikaryon and cell processes (Figs. 2 and 4, $a$ and $c$ ). An accentuation of the reaction product was often observed at the cell periphery (Fig. $2 b$ ). Nuclei remained unstained. Sections of neocortex showed similar neuronal staining (Fig. 4c). In areas such as the spinal anterior horns, the motor neurons were surrounded by small, round densities (Fig. 2c). These profiles, which have the size and distribution of large synaptic terminals, will be described in more detail below, since these reactions remain after paraffin embedding. Reaction product in neuropil appeared as fine, granular material in the neocortex (Fig. 4c) or as larger, more discrete densities and processes, as in the pontine tegmentum and spinal gray (Figs. $2 a$ and $c$, and $4 a$ ).

Axons, observed in either transverse or longitudinal sections, stained homogeneously (Fig. 4c). The sheaths of myelinated fibers did not stain, forming clear halos about the axons.

Immunocytochemical reaction was also observed in astrocytes, particularly those of the white matter. In the subcortical white matter, for example, both cell bodies and cytoplasmic processes of fibrous astrocytes were stained (Fig. 2d). Processes could be traced to their end feet at capillaries. Interfascicular oligodendrocytes of CNS white matter tracts also displayed reaction product, as a thin rim of cytoplasmic staining about an unstained nucleus (not shown). As expected, reaction product was 

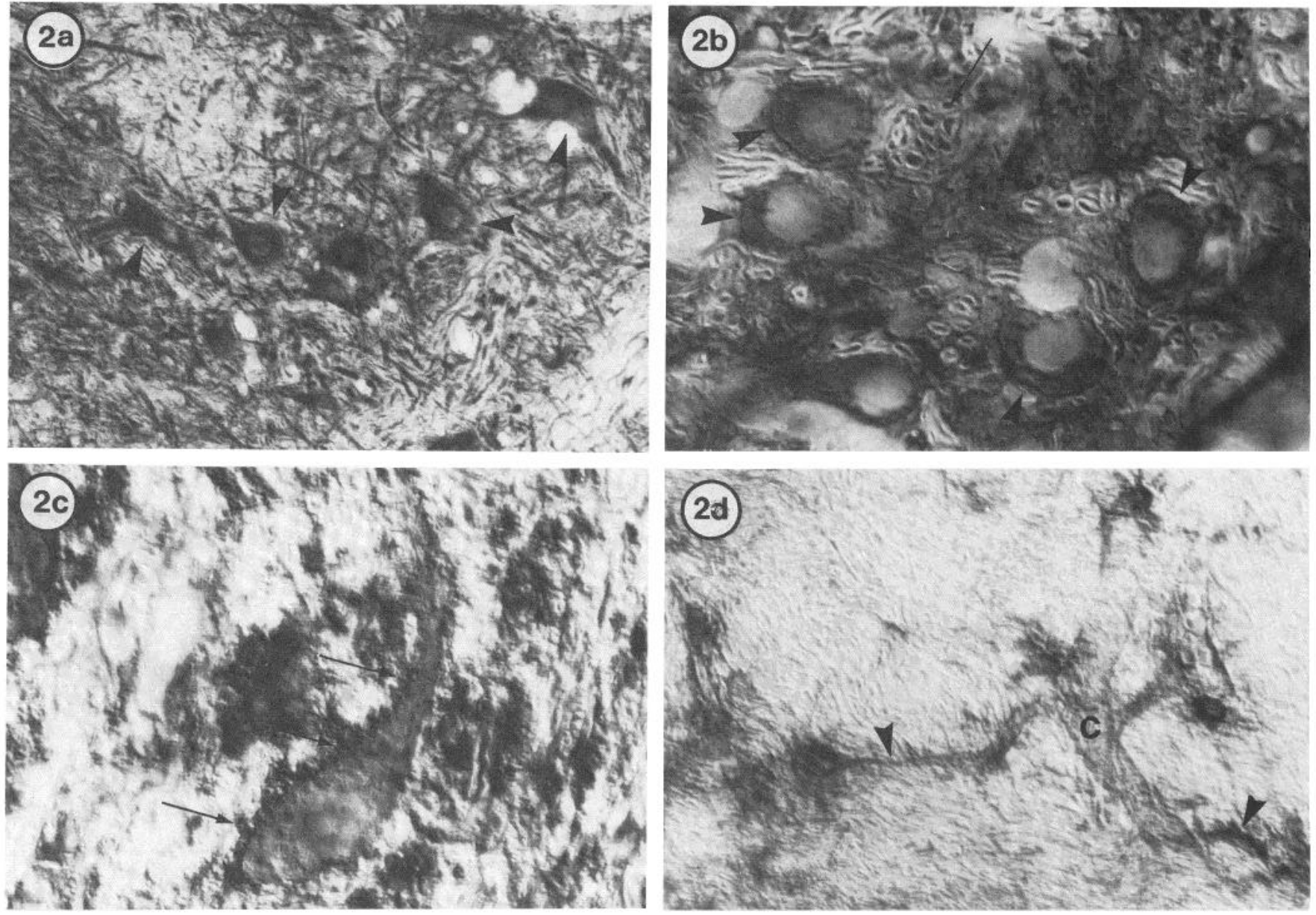

(2e)

Figure 2. Immunocytochemical reactions on Vibratome sections of rat CNS with the anti-actin antiserum. $a$, In the facial nucleus, neurons (arrowheads) and a multitude of thin cell processes show reaction product. Magnification $\times 190$. $b$, Neurons of the lateral vestibular nucleus display a homogeneous cytoplasmic staining with accentuation about the periphery (arrowheads). Axons cut transversely show reaction product (arrow). Magnification $\times 680$ ). $c$, Anterior horn cell shows cytoplasmic staining, as well as discrete densities surrounding perikarya and processes (three shown by arrows). Magnification $\times 660$. $d$, Reaction product appears in astrocytes of the subcortical white matter. Cell processes (arrowheads) can be followed to end feet at capillary $(C)$. Magnification $\times 435$. $e$, Nonimmune rabbit serum yields no immunocytochemical reaction in this section of brainstem. Magnification $\times 170$. All sections were photographed with Nomarski optics.

deposited in the walls of blood vessels, especially the smooth muscle layer of arteries and arterioles (not shown).

Paraffin sections. Immunocytochemistry of paraffin sections showed a far more restricted distribution of the antigen than that seen in Vibratome sections. Possible reasons for this difference will be discussed below. Paraffin sections did not, however, show any new localizations that were not seen in the Vibratome sections. In general, the diffuse cytoplasmic staining of neuronal 
perikarya and processes and of fibrous astrocytes was not evident in paraffin sections. Instead, a nonuniform distribution of reaction product was found. In the spinal gray matter and brainstem tegmentum, for example, the bulk of neuronal cytoplasm was not stained, but particularly dense labeling was present about the perimeters of neurons and as small densities in the neuropil (Figs. 3 and $4 b$ ). When large neurons of the spinal anterior horns and lateral vestibular nucleus were examined at high magnification, the PAP reaction product appeared as discrete, round profiles surrounding and following the outline of cell perikarya. (Fig. $3, b$ and $c$ ). Most of these densities measured between 1 and $4 \mu \mathrm{m}$ in diameter; some appeared more elongated, the long axis apposed closely to the neuron. A large proportion of the somal surface, as well as that of the proximal processes, was covered by these profiles. Occasional densities the size of those at the cell perimeter were seen over cell bodies (see Fig. 3c); these may represent a plane of section just at the top or bottom surface of the neuron. Many similar punctate profiles were observed in the neuropil of the spinal gray and pontine tegmentum (Fig. 3, $a$ to $c$ ). These bore no apparent relationships to neuronal perikarya.

In the cerebellar cortex, clusters of small densities were observed between internal granule neurons (Figs. $3 d)$. Fortuitous sections revealed a rosette like pattern of the profiles, surrounding a less dense core. The size and arrangement of such densities are identical to the morphology of mossy fiber rosettes (Palay and ChanPalay, 1974).

In the neocortex, a diffuse, somewhat granular pattern of reaction product was present, in which neuronal somata and apical dendrites stood out in unstained relief (Fig. 4d). Reaction product was absent in cortical axons and in axons of myelinated fiber tracts in the brainstem and spinal cord (Fig. 4f).

\section{Electron microscopy}

Further investigation of actin localization was performed at the ultrastructural level. Since tissue for electron microscopy was derived from Vibratome sections after immunocytochemical reaction, the results described below can best be compared to the light microscopy of Vibratome sections, rather than that of paraffin sections. Neuronal perikarya and neuropil of the lateral brainstem and the spinal cord were examined. Cytoplasmic staining in neuronal perikarya, while diffuse, did not appear homogeneous. Thus, although reaction product was observed throughout the cytoplasm, the highest density appeared at the periphery of the neuron (Fig. 5a). Postsynaptic sites showed a particularly dense accumulation (see Fig. 7). Large synaptic terminals at the borders of spinal motor neurons contained reaction product (Fig. $5 b$ ). The PAP product had been deposited in synaptic cytoplasm, forming a dense matrix in which vesicles were embedded. A similar, diffuse reaction was present in large dendrites (Fig. 6). The pattern of staining did not follow a distinct, longitudinal course, which would have been expected of other cytoskeletal elements such as neurofilaments and microtubules. The mitochondrial matrix remained unstained, but there was an accentuation about the periphery of mitochondria. As in perikarya, there appeared to be an increase in staining under the plasma membrane (Fig. 6). Small dendritic processes in the neuropil showed a similar subplasmalemmal accumulation at sites where other cell processes were apposed, a number of which contacts could be identified as synapses (Fig. 7).

Astrocytes also displayed a diffuse cytoplasmic staining. Accentuation of the reaction, as in neurons, appeared about mitochondria and underneath the plasma membrane (Fig. 8).

\section{Discussion}

Immunocytochemical reactions of rat CNS with a specific anti-actin antiserum have revealed a widespread distribution of the antigen. In Vibratome sections, reaction product was deposited over neuronal perikarya, excluding nuclei, in axons and in dendrites. Astrocytes and the walls of blood vessels were also stained. Myelin sheaths were not, however. A broad cellular distribution might be expected, inasmuch as the CNS is a rich source of actin (see, e.g., Bray and Thomas, 1976). Furthermore, actin is a component of most, if not all, cells. Previous immunofluorescent studies of the nervous system with anti-actin antisera have demonstrated cytoplasmic staining of astrocytes (Groschel-Stewart et al., 1977) and blood vessels (Owman et al., 1978). Although neuronal staining was not noted in these reports, other studies have described immunocytochemical reactions in mossy fiber terminals in the cerebellar cortex (Toh el al., 1976), appearing much like those shown in Figure $3 d$ ).

Under the conditions of fixation and tissue processing used for Vibratome sections, the neuronal cytoplasm in perikarya, axons, and dendrites contained reaction product. The cytoplasmic reactions observed in Vibratome sections appeared specific for actin. There was no reaction with nonimmune serum or antiserum adsorbed with actin; furthermore, treatment of sections with sodium borohydride did not alter the pattern of reaction. These sets of controls argue against nonspecific binding of immunoglobulins to sections. At the light microscopic level, reaction appeared as a homogeneous staining, with a peripheral accentuation. At the electron microscopic level, reaction product was distributed throughout the cytoplasm, whether in perikarya or processes. The pattern of staining did not follow that of well defined organelles, however, such as mitochondria, neuronal or glial intermediate filaments, microtubules, or smooth membranes. Accentuation about mitochondria and microtubules appeared in several sections (Figs. 6 and 7).

There were regions in which reaction product appeared especially concentrated. In general, the subplasmalemmal zone displayed a denser reaction than the bulk of the cytoplasm. Previous ultrastructural work, as well as a large literature on actin in cultured cells (see, e.g., Chang and Goldman, 1973; Schloss et al., 1977; Willingham et al., 1981), would suggest that much of the subplasmalemmal reaction coincides with a dense network of microfilaments. Reaction product in postsynaptic zones, either on neuronal perikarya or on processes in the neuropil, was especially concentrated at the site of contact, at a point corresponding to the postsynaptic density. This finding provides direct evidence for an 

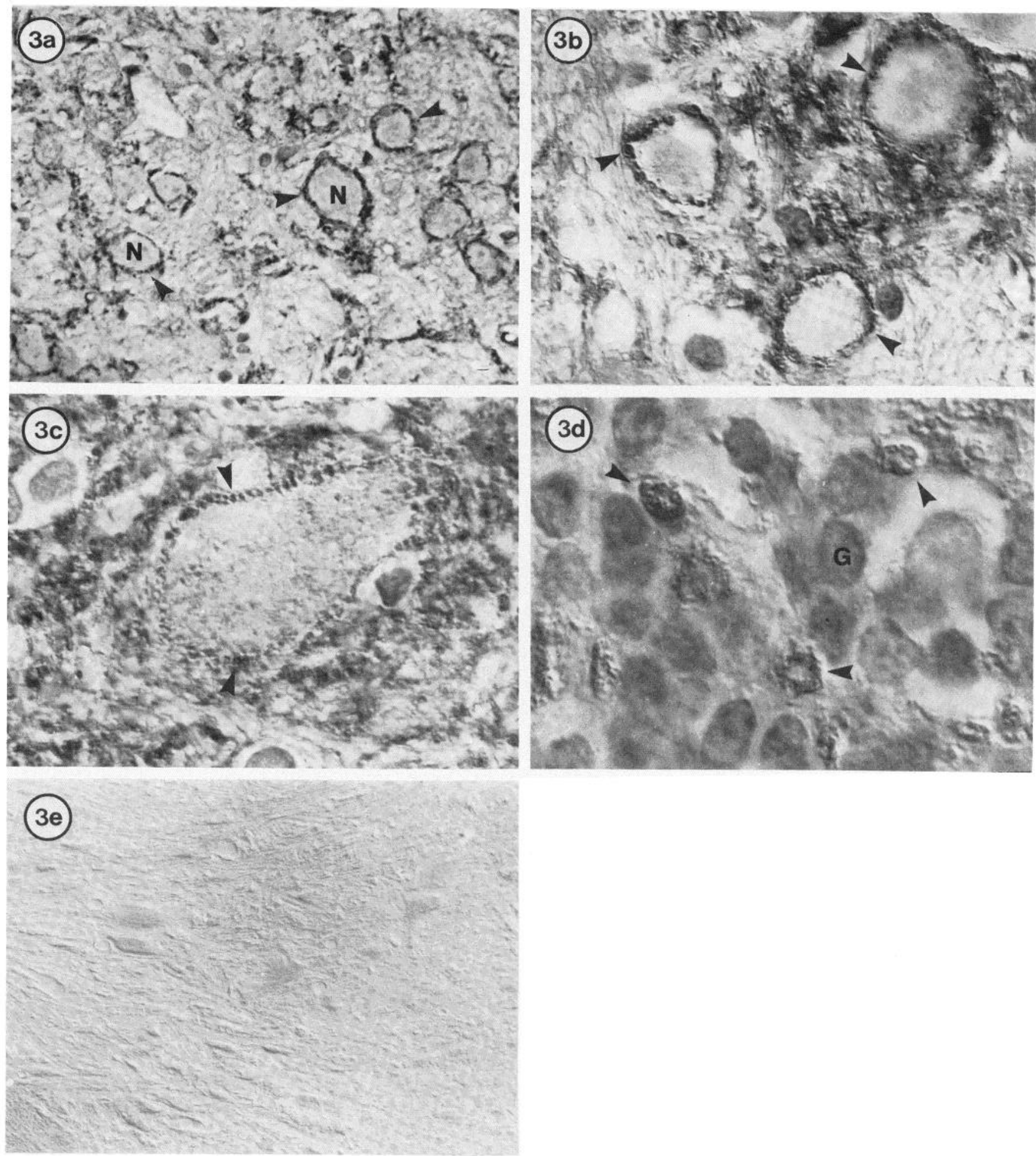

Figure 3. Immunocytochemical reactions on paraffin sections of rat CNS with the anti-actin antiserum. $a$, In the lateral vestibular nucleus, the dark reaction product (examples marked with arrowheads) is deposited about the periphery of neurons $(N)$. Magnification $\times 270$. $b$, At higher magnification, the densities about vestibular neurons appear discrete and rounded, covering much of the somal surface (arrowheads). Similar punctate profiles are present in the neuropil between cell bodies. Magnification $\times 1000 . c$, Reaction product in discrete profiles (arrowheads) surrounds the perikaryon of an anterior horn motor neuron. Magnification $\times 1000$. $d$, In the cerebellar granule cell layer, clusters of PAP product are present between granule cells $(G)$. Several display a rosette-like pattern, with rounded densities surrounding a lighter staining core (arrowheads). Magnification $\times 1800$. $e$, Anti-actin antiserum, adsorbed with rat brain actin, yields no cytochemical reaction in this section of brainstem. Magnification $\times 230$. All sections were counterstained with hematoxylin, which provides a nuclear stain, and $b$ to $e$ were photographed with Nomarski optics. 

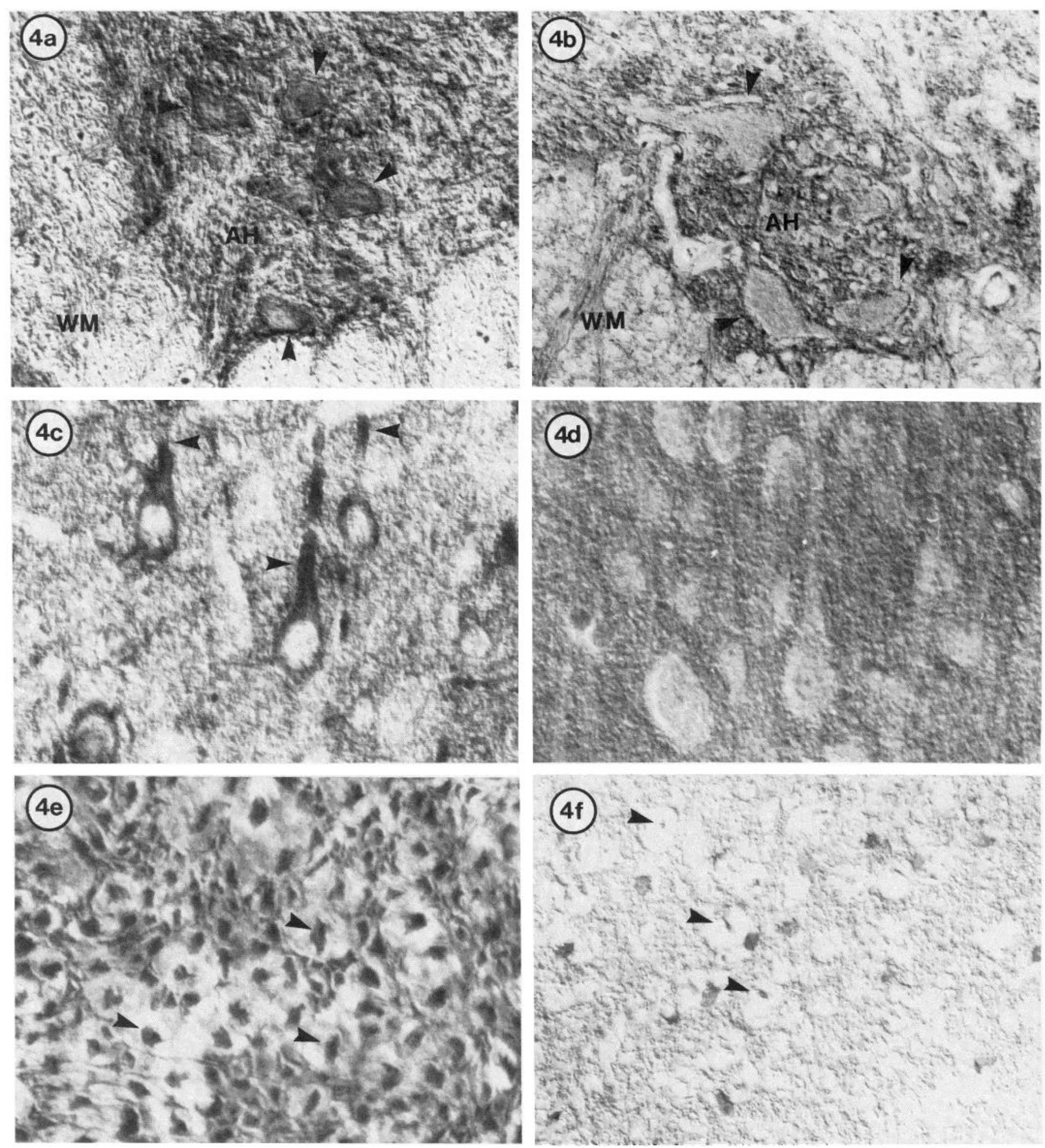

Figure 4. Comparisons of immunocytochemical reactions on Vibratome and paraffin sections. $a$, In this Vibratome section of spinal cord anterior horn $(A H)$, reaction product is seen in neurons (arrowheads) and cell processes. Accentuation is seen at the periphery of neurons. $W M$, white matter. Magnification $\times 190$. $b$, In a paraffin section of anterior horn $(A H)$, there is little cytoplasmic staining in neuronal perikarya (arrowheads). Reaction product remains with densities about motor neurons and in neuropil. Magnification $\times 190$. $c$, This Vibratome section of neocortex shows cytoplasmic staining of neuronal perikarya and dendrites (arrowheads). Magnification $\times 430$. $d$, In this paraffin section of neocortex, little cytoplasmic reaction remains. A granular staining pattern of the neuropil is seen. Counterstained with hematoxylin. Magnification $\times 530$. $e$, Axons in Vibratome section of the spinal posterior columns appear as dense profiles surrounded by unstained myelin sheaths. Magnification $\times 820$. $f$, Axonal staining has disappeared after paraffin embedding. Axons and myelin are illustrated by arrowheads. Stained profiles are astrocyte nuclei; counterstained with hematoxylin. Magnification $\times 820$. 

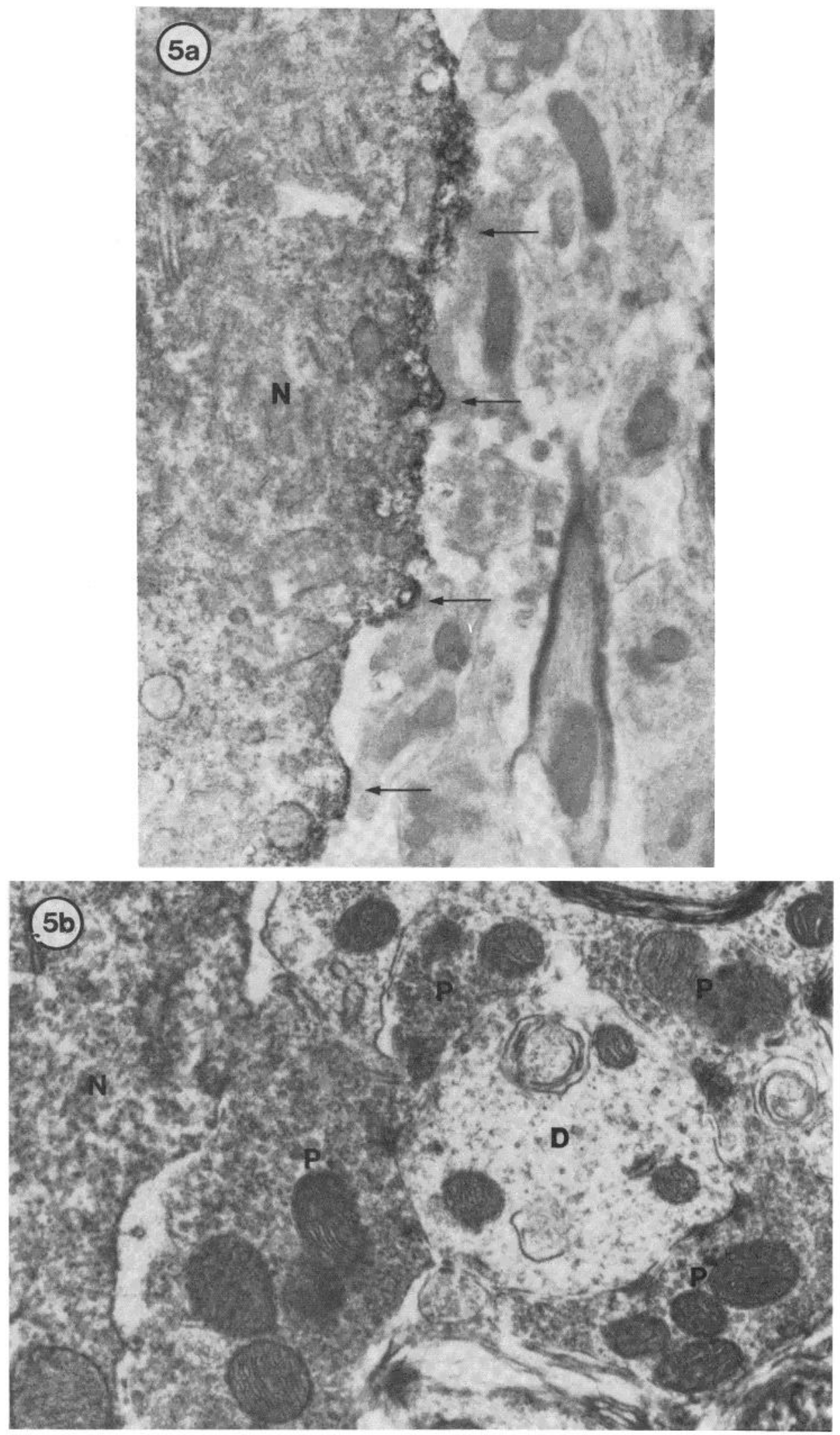

Figure 5. Immunocytochemical reaction at the ultrastructural level. $a$, Neuron $(N)$ in the lateral vestibular nucleus contains reaction product over the cytoplasm, with densest labeling at the cell surface. The heaviest accumulations are at sices where other (unlabeled) cell processes are apposed to the neuron (arrows). Magnification $\times 15,000 . b$, In the spinal anterior horn, four labeled terminals $(P)$ contact a dendrite $(D)$ (unlabeled) near a motor neuron cell body $(N)$. The section was stained briefly with uranyl acetate. Magnification $\times 33,350$. 


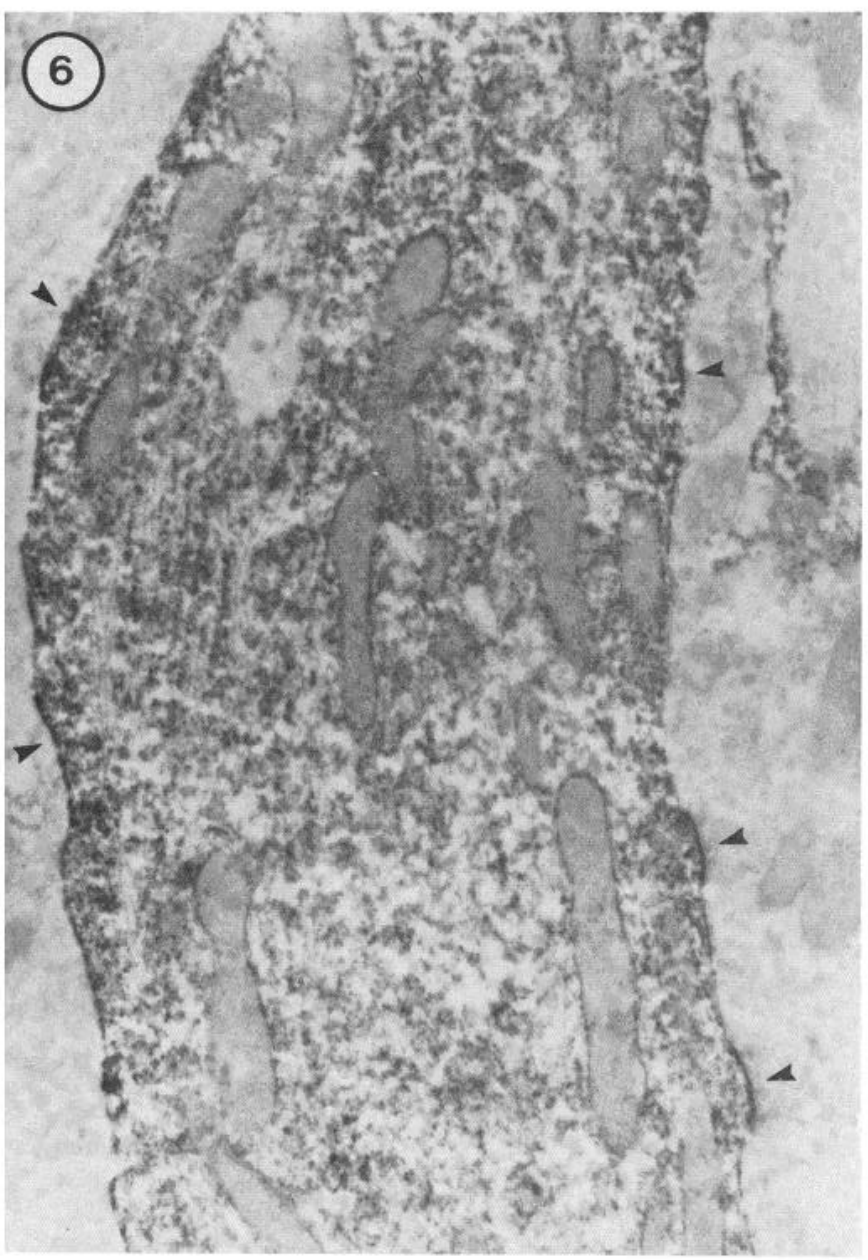

Figure 6. A large proximal dendrite in the spinal anterior horn contains reaction product throughout the cytoplasm, with densest labeling at the plasma membrane (examples illustrated by arrowheads). Magnification $\times 19,400$.

association of actin with synaptic densities and supports biochemical studies which show actin as a major component of postsynaptic density fractions (Cohen et al., 1977; Kelly and Cotman, 1978; Matus and Taff-Jones, 1978). It also supports recent freeze etch studies of the guinea pig cochlear nucleus (Gulley and Reese, 1981), in which the microfilaments projecting into the postsynaptic cytoplasm from an attachment within the postsynaptic membrane were tentatively identified as polymerized actin, and myosin subfragment-binding studies, which show actin filaments attached to postsynaptic zones (Fifkova and Delay, 1982). ${ }^{2}$

The accumulation of actin in association with the postsynaptic membrane suggests an analogy with non-

\footnotetext{
${ }^{2}$ While this manuscript was under review, Matus et al. (1982) reported immunocytochemical reactivity with an anti-actin antiserum of postsynaptic areas, particularly the postsynaptic density, and blood vessel walls of the rat CNS. The staining pattern appears similar to that observed with paraffin sections in the present study; i.e., synaptic regions are densely labeled, but there is little general cytoplasmic reaction in neurons or glia. Variations in antibody titers, and method ological differences, probably coupled with differential fixation or extraction of actin in its various organizational forms, may explain apparent differences in immunocytochemical localization, as discussed below.
}

neuronal cells, in which clustering of membrane proteins is associated with subplasmalemmal concentrations of actin (Ash et al., 1977; Gabbiani et al., 1977; Toh and Hard, 1977; Bourguignon and Rosek, 1980; Hall et al., 1981). Linkages of actin to membrane proteins, either directly or through intermediates, has been demonstrated (Flanagan and Koch, 1978). Thus, a number of investigators have suggested that cytoskeletal elements beneath, and linked to, cell membranes play an important role in determining the distribution of certain membrane proteins. In CNS neurons there is evidence that receptors to neurotransmitters are clusted at discrete sites (Ransom et al., 1977; Choi and Fischbach, 1981). In parasympathetic neurons, high acetylcholine receptor densities have been found at synaptic terminals on perikarya (Harris et al., 1971). A concordance of transmitter receptors with underlying cytoskeletal proteins would suggest that neurons employ mechanisms common to many cells to produce nonuniform distributions of membrane proteins.

The diffuse intraneuronal staining indicated that a significant proportion of the neuronal actin is found in the cytoplasm, in addition to that associated with the plasma membrane. This widespread localization is consistent with the idea that actin forms part of a cytoplasmic network of filamentous structures (see, e.g., Lasek and Shelanski, 1981). The technique employed in this report, however, presumably does not distinguish between monomeric and filamentous actin. Thus, without further studies, it is not possible to identify particular sites of reaction with specific states of actin organization. Probes specific for F-actin, and immunocytochemical methods which yield smaller reaction products than the relatively coarse PAP product, such as colloidal gold (Roth et al., 1978) or ferritin (Willingham et al., 1981), will be useful in helping to probe different states of contractile protein organization in neurons.

The sporadic nature of the staining in electron micrographs, with negative neuronal and astrocytic processes next to positive ones, most likely reflects a nonuniform penetration of reagents into the tissue sections, rather than an absence of antigen in a large number of cells. Cutting Vibratome sections transversely revealed that the PAP reaction penetrated only 4 to $5 \mu \mathrm{m}$ into the tissue, with fewer positive elements seen as deeper levels were examined. Neuronal and glial processes deep in the section that contain reaction product are probably connected to cell bodies or processes near to or exposed at the face of the section.

The pattern of immunocytochemical staining in paraffin sections appeared more restricted than that in Vibratome sections. Much of the reaction in neuronal cell bodies, axons, and dendrites was not evident. The remaining reaction product appeared discretely localized. Observations of large neurons in the spinal anterior horn and lateral vestibular nucleus and of the cerebellar granule cell layer suggested that high levels of actin were found at synaptic junctions. The size and pattern of distribution of the densities appear similar to that of the boutons on cat spinal motor neurons visualized by silver stains (a number of studies are summarized in Illis, 1964) and are also consistent with ultrastructural studies, which have demonstrated that large synaptic boutons 


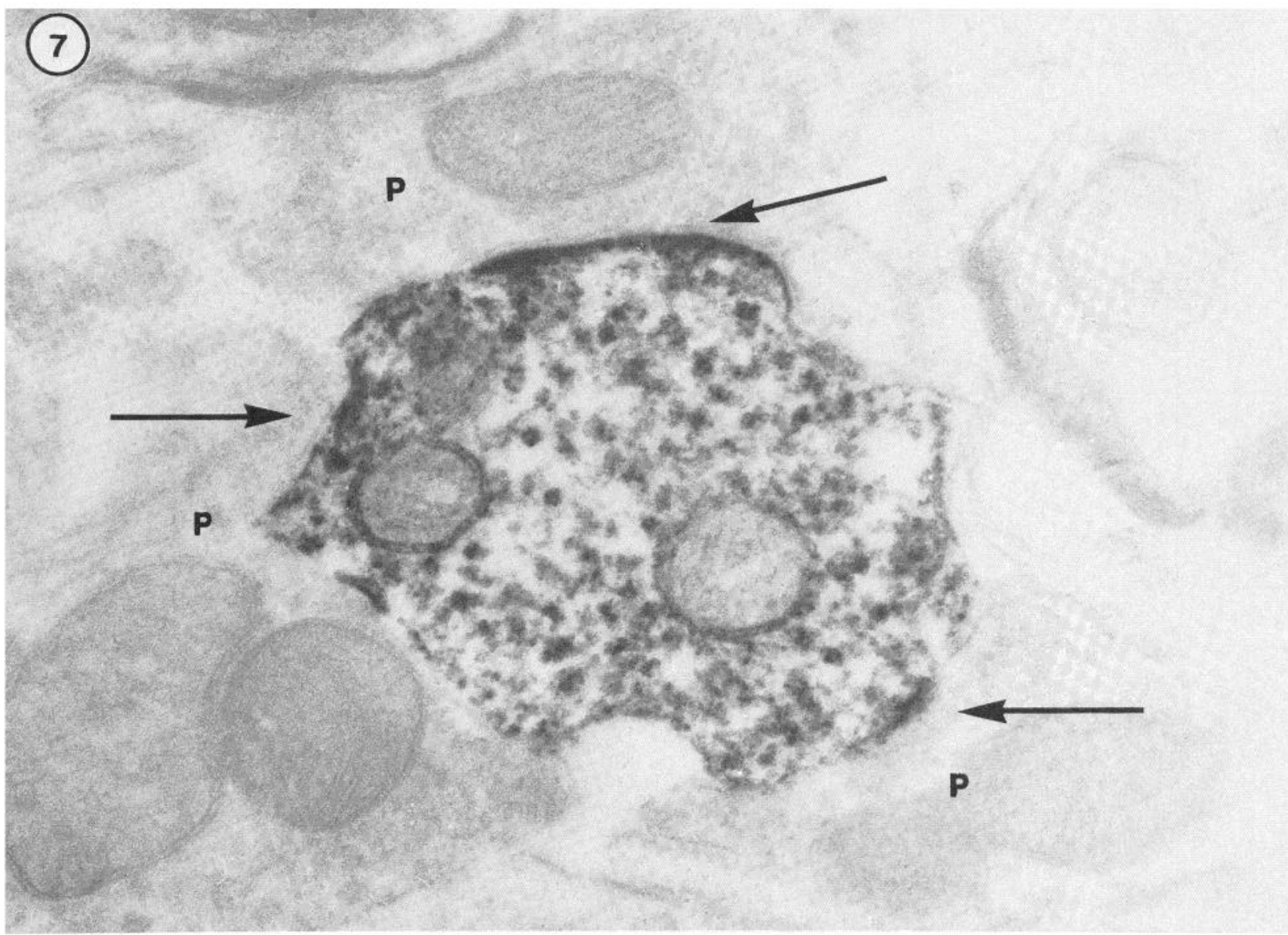

Figure 7. A small process in the neuropil, probably dendritic, shows postsynaptic labeling (arrows). $P$, presynaptic terminals. Magnification $\times 54,000$.

cover a substantial proportion of the perikaryal surface of anterior horn cells and vestibular neurons (Bodian, 1964, 1966; Conradi, 1969; Sotelo and Palay, 1970; McLaughlin, 1972). In contrast to these areas of the CNS, where large synapses are prominent, the cerebral cortex, where the neuropil contains a myriad of smaller synaptic contacts, displayed a granular staining pattern, in which individual synapses could not be resolved.

The principal disparity between the Vibratome and paraffin sections was that, in the latter, the bulk of cytoplasmic reaction had disappeared. Reaction product at synaptic terminals seemed to remain, judging by the size and distribution of the positive densities. Whether the postsynaptic staining also remained is difficult to say at the light microscopic level. Two general reasons may obtain to explain this methodological difference. First, tissue processing for paraffin sections may alter antigen reactivity of the bulk of cytoplasmic actin, rendering it unrecognizable by the antiserum. Second, the processing may extract actin from the tissue. Extraction could either be a nonselective process, in which case embedding depletes actin in all locations, or selective, in which case actin in certain locations only would be removed. If a nonselective extraction were to occur, then the synaptic reaction would reflect a higher initial concentration of the antigen. Selective extraction could reflect different states of actin organization in various regions of the neuron, each with different sensitivities to technical procedures. Actin exists in both monomeric and polymeric forms in the CNS and in other tissues (see, e.g., Weeds, 1982). Polymers may be further organized by a variety of accessory proteins, the binding of which could confer extra stability to microfilaments.

The diminution of cytoplasmic staining in paraffin sections appears reproducible, in that it has been observed in a number of rat brains used in this study, as well as in mouse and human CNS (unpublished observations). Furthermore, under some conditions, dense cytoplasmic staining is retained in paraffin sections: in neurons migrating during development, in neurons involved in new dendritic growth, and in abnormal, filamentous inclusions within neurons (unpublished observations; Goldman, 1982). The positive cytoplasmic reaction in these cases may indicate that in certain stages of development or during pathological conditions, neurons may organize large proportions of their cytoplasmic actin into more stable forms.

Immunocytochemical studies with actin antisera, particularly applied to paraffin sections, have several potential applications for further study of synaptic organization. At the light microscopic level, the pattern of synaptic input over a wide field can be visualized, encompassing many neurons. Thus, immunocytochemistry may be useful in studying patterns of innervation during 


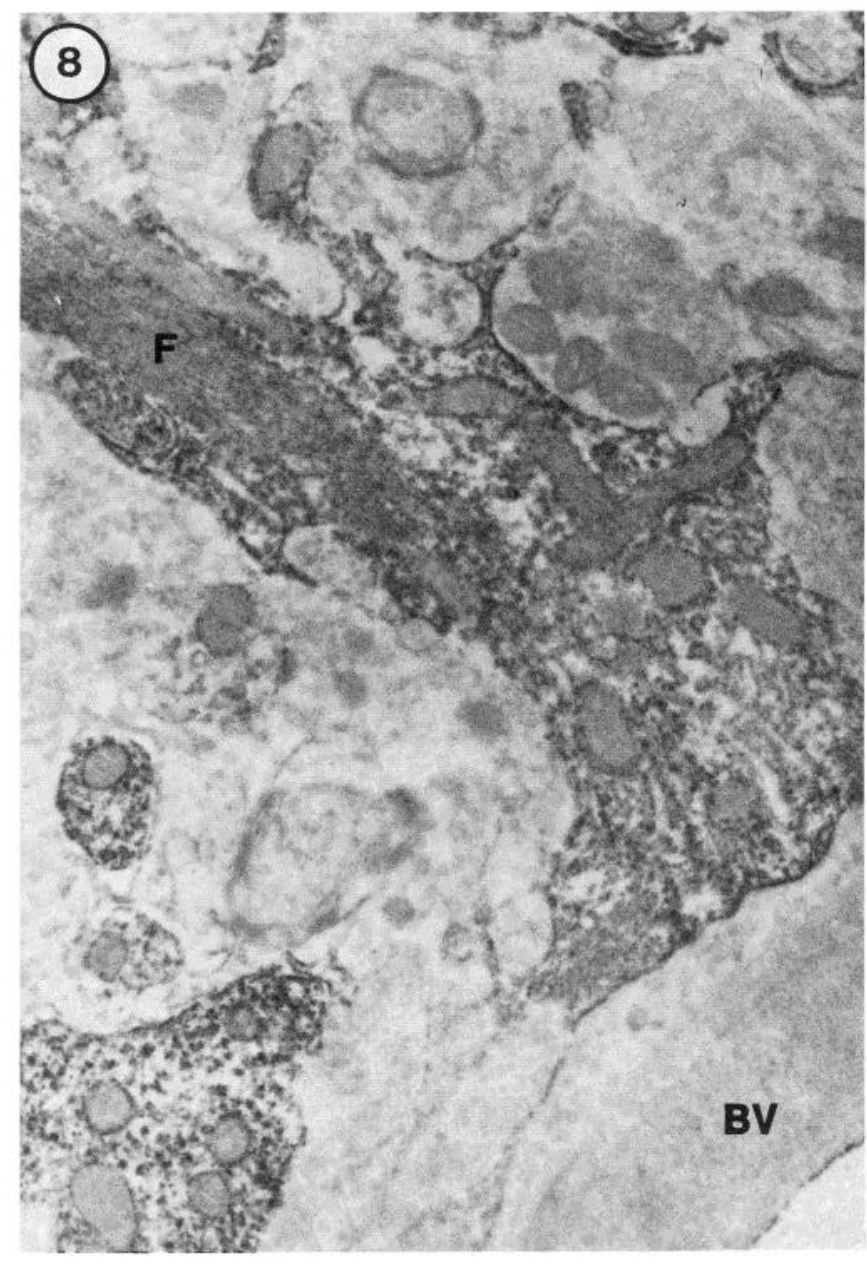

Figure 8. An astrocyte process containing a bundle of intermediate filaments $(F)$, an end foot terminating at a blood vessel $(B V)$, and thin cytoplasmic processes displays cytoplasmic labeling, also with some accumulation at the plasma membrane. Magnification $\times 7700$.

development or under pathological conditions. It would be of interest, for example, to examine whether clustering of actin beneath the membrane of central neurons occurs before innervation or changes during conditions such as denervation or chromatolysis. This technique is, of course, not selective for transmitter type, but could be used in conjunction with antisera to specific neurotransmitters (see, e.g., Ruda, 1982) to assess patterns of specific innervation.

\section{References}

Alonso, G., F. Gabrion, E. Travers, and I. Assenmacher (1981) Ultrastructural organization of actin filaments in neurosecretory axons of the rat. Cell Tissue Res. 214: 323-341.

Ash, J. F., D. Louvard, and S. J. Singer (1977) Antibodyinduced linkages of plasma membrane proteins to intracellular actomyosin in cultured fibroblasts. Proc. Natl. Acad. Sci. U. S. A. 74: 5584-5588.

Blitz, A., and R. Fine (1974) Muscle-like contractile proteins and tubulin in synaptosomes. Proc. Natl. Acad. Sci. U. S. A. 71: 4472-4476.

Bodian, D. (1964) An electron microscopic study of the monkey spinal cord. Bull. Johns Hopkins Hosp. 114: 13-119.

Bodian, D. (1966) Synaptic types on spinal motoneurons: An electron microscopic study. Bull. Johns Hopkins Hosp. 119: $16-45$.

Bourguignon, L. Y. W., and R. J. Rosek (1980) Capping of concanavalin A receptors and their association with microfilaments in monolayer grown human fibroblastoid cells. Cell Tissue Res. 205: 77-84.

Bray, D., and C. Thomas (1976) Unpolymerized actin in fibroblasts and brain. J. Mol. Biol. 105: 527-544.

Chang, C. M., and R. D. Goldman (1973) The localization of actin-like fibers in cultured neuroblastoma cells as revealed by heavy meromyosin binding. J. Cell Biol. 57: 867-874.

Chiu, F. -C., W. T. Norton, and K. L. Fields (1981) The cytoskeleton of primary astrocytes in culture contains actin, glial fibrillary acidic protein, and the fibroblast-type intermediate filament protein, vimentin. J. Neurochem. 37: 147155.

Choi, D. W., and G. D. Fischbach (1981) GABA conductance of chick spinal cord and dorsal root ganglia neurons in cell culture. J. Neurophysiol. 45: 605-620.

Cohen, R. S., F. Blomberg, K. Borzins, and P. Siekevitz (1977) The structure of postsynaptic densities isolated from dog cerebral cortex. I. Overall morphology and protein composition. J. Cell Biol. 74: 181-203.

Conradi, S. (1969) On motoneuron synaptology in adult cats. Acta Physiol. Scand. Suppl. p. 332.

Fifkova, E., and R. J. Delay (1982) Cytoplasmic actin in neuronal processes as a possible mediator of synaptic plasticity. J. Cell Biol. 95: 345-350.

Flanagan, J., and G. L. E. Koch (1978) Cross-linked surface Ig attaches to actin. Nature 273: 278-281.

Gabbiani, G., C. Chapponnier, A. Zumbe, and P. Vassalli (1977) Actin and tubulin co-cap with surface immunoglobulins in mouse B lymphocytes. Nature 269: 697-698.

Goldman, J. E. (1982) The association of actin with Hirano bodies. J. Neuropathol. Exp. Neurol. 42: 146-152.

Goldman, J. E., and F. -C. Chiu (1983) Growth kinetics, cell shape, and the cytoskeleton of primary astrocyte cultures. J. Neurochem., in press.

Goldman, J. E., H. H. Schaumburg, and W. T. Norton (1978) Isolation and characterization of glial filaments from human brain. J. Cell Biol. 78: 426-440.

Groschel-Stewart, U., K. Unsicker, and H. Leonhardt (1977) Immunohistochemical demonstration of contractile proteins in astrocytes, marginal glia, and ependymal cells in rat diencephalon. Cell Tissue Res. 180: 133-137.

Gulley, R. L., and T. S. Reese (1981) Cytoskeletal organization at the postsynaptic junction. J. Cell Biol. 91: 298-302.

Hall, Z. W., B. W. Lubit, and J. H. Schwartz (1981) Cytoplasmic actin in postsynaptic structures at the neuromuscular junction. J. Cell Biol. 90: 789-792.

Harris, A. J., S. W. Kuffler, and M. J. Dennis (1971) Differential chemosensitivity of synaptic and extrasynaptic areas on the neuronal surface membrane in parasympathetic neurons of the frog, tested by microapplication of acetylcholine. Proc. R. Soc. Lond. Biol. 177: 541-553.

Herman, I. M., and T. D. Pollard (1979) Comparison of purified anti-actin and fluorescent heavy meromyosin staining patterns in dividing cells. J. Cell Biol. 80: 509-520.

Illis, L. (1964) Spinal cord synapses in the cat: The normal appearances by the light microscope. Brain 87: 543-553.

Kelly, P. T., and C. W. Cotman (1978) Synaptic proteins: Characterization of tubulin and actin and identification of a distinct postsynaptic density polypeptide. J. Cell Biol. 79: 173-183.

Lasek, R. J., and M. L. Shelanski (eds.) (1981) Cytoskeletons and the Architecture of Nervous Systems. Neurosci. Res. Program Bull. 19: 1-152.

LeBeaux, Y. J., and J. Willemot (1975) An ultrastructural study 
of the microfilaments in rat brain by means of E-PTA staining and heavy meromyosin labeling. II. The synapses. Cell Tissue Res. 160: 37-68.

Lindberg, U., and S. Eriksson (1971) Purification from crude extract by affinity chromatography of the inhibitor of deoxyribonuclease I. Eur. J. Biochem. 18: 474-479.

Marotta, C., P. Strocchi, and J. M. Gilbert (1978) Microheterogeneity of brain cytoplasmic and synaptoplasmic actins. J. Neurochem. 30: 1441-1451.

Matus, A. I., and D. H. Taff-Jones (1978) Morphology and molecular composition of isolated postsynaptic junctional structures. Proc. R. Soc. Lond. Biol. 203: 135-151.

Matus, A. I., M. Ackermann, G. Pehling, H. R. Byers, and K. Fujiwara (1982) High actin concentrations in brain dendritic spines and postsynaptic densities. Proc. Natl. Acad. Sci. U. S. A. 79: 7590-7594.

McLaughlin, B. J. (1972) Dorsal root projections to the motor nuclei in the cat spinal cord. J. Comp. Neurol. 144: 461-474.

Metuzals, J., and W. E. Mushynski (1974) Electron microscope and experimental investigation of the neurofilamentous network of Dieter's neurons. J. Cell Biol. 61: 701-722.

Owman, C., L. Edvinsson, J. E. Hardebo, U. Groschel-Stewart, K. Unsicker, and R. Walles (1978) Immunohistochemical demonstration of actin and myosin in brain capillaries. Adv. Neurol. 20: 35-37.

Palay, S. F., and V. Chan-Palay (1974) Cerebellar Cortex, Springer-Verlag, New York.

Puszkin, S., and S. Berl (1972) Actomyosin-like protein from brain. Biochim. Biophys. Acta 256: 695-709.

Puszkin, S., W. J. Nicklas, and S. Berl (1972) Actomyosin-like protein in brain: Subcellular distribution. J. Neurochem. 19: 1319-1333.
Ransom, B. R., C. N. Christian, P. N., Bullock, and P. G. Nelson (1977) Mouse spinal cord in cell culture. III. Neuronal chemosensitivity and its relationship to synaptic activity. J. Neurophysiol. 40: 1163-1176.

Roth, J., M. Bendayan, and L. Orci (1978) Ultrastructural localization of intracellular antigens by the use of a Protein A-gold complex. J. Histochem. Cytochem. 26: 1074-1081.

Ruda, M. A. (1982) Opiates and pain pathways: Demonstration of encephalin synapses on dorsal horn projection neurons. Science 215: 1523-1525.

Schloss, J. A., A. Milsted, and R. D. Goldman (1977) Myosin subfragment binding for the localization of actin-like microfilaments in cultured cells. A light and electron microscopic study. J. Cell Biol. 74: 794-815.

Sotelo, C., and S. F. Palay (1970) The fine structure of the lateral vestibular nucleus in the rat. II. Synaptic organization. Brain Res. 18: 93-115.

Sternherger, I. A. (1979) Immunocytochemistry, Fd. 2, John Wiley \& Sons, Inc., New York.

Toh, B. H., and G. C. Hard (1977) Actin co-caps with concanavalin A receptors. Nature 269: 695-697.

Toh, B. H., H. A. Gallichio, P. L. Jeffrey, B. G. Livett, H. K. Muller, M. N. Cauchi, and F. M. Clarke (1976) Anti-actin stains synapses. Nature 264: 648-650.

Weeds, A. (1982) Actin-binding proteins-Regulators of cell architecture and motility. Nature 296: 811-816.

Willingham, M. C., S. S. Yamada, P. J. A. Davies, A. V. Rutherford, M. G. Gallo, and I. Pastan (1981) Intracellular localization of actin in cultured fibroblasts by electronmicroscopic immunocytochemistry. J. Histochem. Cytochem. 29: $17-37$. 